NOTE TECHNIQUE

\title{
APPLICATION DE L'HYDROACOUSTIQUE EN MILIEU FLUVIAL : EXEMPLE DE L'ÉTUDE DE LA MONTAISON DU SAUMON ATLANTIQUE (SALMO SALAR L.) PAR SONDAGE A DOUBLE FAISCEAU DANS LA RIVIĖRE MOISIE, QUÉBEC.
}

\author{
F. LÉVESQUE, M.K. HARTE * \\ Groupe Environnement Shooner inc., \\ 5355, boul. des Gradins, bureau 101, Québec (Québec) G2J 1C8, Canada.
}

\begin{abstract}
RÉSUMÉ
Des recherches en hydroacoustique ont été conduites en 1990 et 1991, afin d'étudier la migration du saumon atlantique (Salmo salar) au stade d'adulte dans la rivière Moisie. La méthode employée est celle de sondage à double faisceau, en couple. Les résultats montrent que la majorité des saumons atlantiques nagent près du lit de la rivière à de courtes distances du rivage, dans une zone où les vitesses d'écoulement sont inférieures à environ $1 \mathrm{~m} / \mathrm{s}$. Les décomptes acoustiques de poissons migrant vers l'amont sont significativement corrélés avec les captures de saumons atlantiques au filet piège $(P<0,005)$. Les observations indiquent que la recherche d'un site d'échantillonnage approprié et la connaissance du comportement du poisson, sont deux étapes essentielles à satisfaire pour assurer la réussite d'une opération de suivi des stocks de poissons migrateurs avec l'usage de l'hydroacoustique. Les données de sondage par écho doivent être validées avec d'autres techniques qui permettent d'établir la composition des espèces présentes et d'évaluer la précision de la méthode hydroacoustique employée.
\end{abstract}

\section{PRACTICAL APPLICATION OF HYDROACOUSTIC SENSING IN RIVERS : THE CASE STUDY OF THE ATLANTIC SALMON (SALMO SALAR L.) SPAWNING RUN AS INVESTIGATED BY DUAL-BEAM ECHOSOUNDING IN THE MOISIE RIVER, QUEBEC.}

\begin{abstract}
Hydroacoustic experiments were conducted in 1990 and 1991 in order to assess the adult Atlantic salmon (Salmo salar) run on the Moisie River. The method used was the dualdual beam echosounding. Results indicate that most salmons swim near the river bottom, close to the shores, where flow speed is less than about $1 \mathrm{~m} / \mathrm{s}$. Acoustic counts of fish migrating upstream shows a significant correlation with salmon catches in trap-nets $(P<0,005)$. Our observations indicate that the selection of an adequate sampling site and a good knowledge of fish behaviour are two essential steps to provide for a successful hydroacoustic monitoring of migrating fish stocks. The data from echosounding must be validated by other methods which allow to determine species composition and to assess the accuracy of the hydroacoustic method used.
\end{abstract}

* Adresse actuelle : International Fisheries Investigations - 6031 Woodland PI. N. - Seattle, WA 98103, U.S.A 


\section{INTRODUCTION}

L'hydroacoustique est une technique relativement récente qui présente un grand potentiel dans le domaine de la recherche et de la gestion des stocks de poissons (THORNE, 1983). Elle trouve sa place parmi les techniques modernes d'évaluation des ressources aquatiques parce qu'elle fournit une estimation juste et rapide des changements dans les stocks (MATHISEN, 1980). L'étude de la migration des poissons en rivière, au moyen de transducteurs à double faisceau, s'est développée au cours de la dernière décennie et a porté principalement sur les saumons du Pacifique. Depuis les premiers essais réalisés au milieu des années 1980 (BERNING et al., 1987 ; HARTE et JOHNSTON, 1989) des progrès considérables ont été enregistrés avec cette technique pour évaluer les stocks de poissons migrateurs.

Les remontées de saumons atlantiques (Salmo salar) dans la rivière Moisie, au Québec, ont été étudiées par sondage à double faisceau afin d'évaluer la taille du stock, en 1990 et 1991. Le présent article livre les principaux résultats observés sur l'application de la méthode hydroacoustique utilisée dans le cadre de cette étude. L'objectif est d'analyser les limites de cette méthode, ainsi que les éléments techniques à considérer lorsqu'elle est employée en milieu fluvial.

\section{MATÉRIEL ET MÉTHODES}

La rivière Moisie $\left(50^{\circ} 12^{\prime} \mathrm{N}-66^{\circ} 04^{\prime} \mathrm{O}\right)$ est un grand cours d'eau de la rive nord du fleuve Saint-Laurent (fig. 1), dont le module de débit annuel est de $436 \mathrm{~m}^{3 / \mathrm{s}}$ et le débit moyen estival dépasse les $300 \mathrm{~m}^{3} / \mathrm{s}$. Le cours principal de la rivière est accessible au saumon atlantique sur $262 \mathrm{~km}$. Le site d'étude se trouve à $50 \mathrm{~km}$ de l'embouchure. II a été sélectionné en fonction des critères requis pour utiliser l'hydroacoustique lorsque les transducteurs sont orientés perpendiculairement à la rive, en projection horizontale. Ces critères sont un lit en pente douce qui s'étend jusqu'au point le plus profond situé dans le chenal de la rivière et un écoulement de type laminaire.

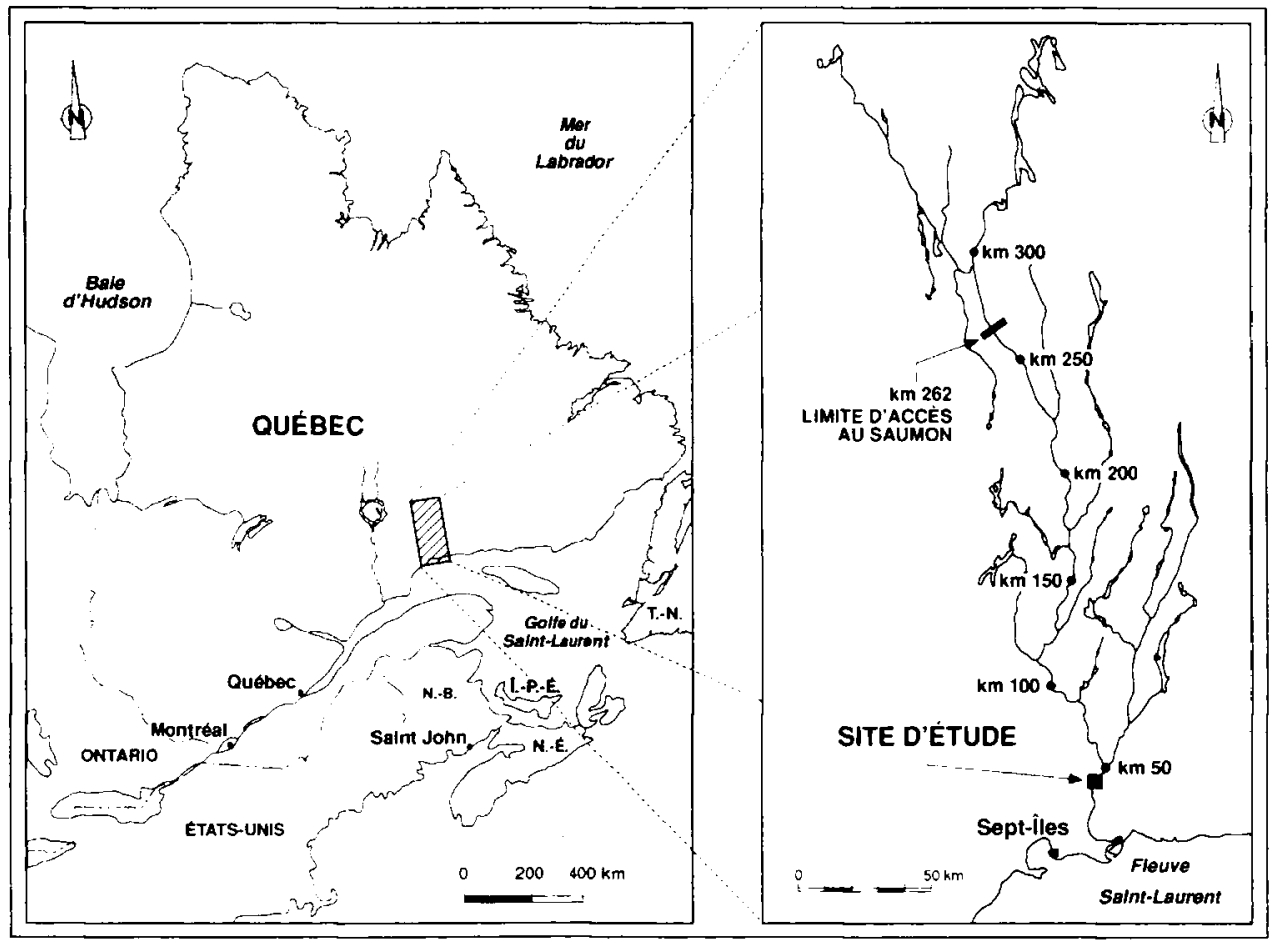

FIGL:RE I

Figure 1 : Localisation du site d'étude hydroacoustique sur la rivière Moisie, Québec. Figure 1 : Location of the hydroacoustic study site on the Moisie River, Quebec. 
L'hydroacoustique consiste à mesurer les retours d'échos des poissons qui traversent un champ d'insonification généré par un sondeur à écho dans un volume d'eau donné. La technique employée dans la rivière Moisie est celle à double faisceaux, en couple. Deux transducteurs produisant chacun deux faisceaux, l'un large et l'autre étroit, sont fixés côte à côte (fig. 2). Les faisceaux doublés d'un même transducteur permettent de détecter chacune des cibles et d'en mesurer la taille acoustique (EHRENBERG, $1982 ; 1984$ ). En établissant lequel des deux transducteurs reçoit un premier écho provenant d'un poisson (au temps $t$ ), et lequel en reçoit le dernier (au temps $t+x$ ), le sens du déplacement peut être déterminé. Cette technique a été utilisée avec succès par HARTE et JOHNSTON (1989).

Durant toute la période d'étude, l'échantillonnage a toujours couvert entièrement la largeur de la rivière, quels que soient les niveaux d'eau. En 1990, les travaux ont porté principalement sur la mise au point d'une stratégie d'échantillonnage. En 1991, deux couples de transducteurs ont été installés de chaque côté de la rivière (fig. $3 a$ ). Le champ des faisceaux sonores fut déployé juste au-dessus du lit. La zone non couverte par les faisceaux était fermée par la présence de clôtures disposées sur chaque rive, en aval des transducteurs, qui contribuaient aussi à diriger les poissons dans le champ d'insonification efficace des faisceaux (fig. 3b).

Les équipements hydroacoustiques utilisés au cours des deux saisons étaient les mêmes, à la seule différence que le sondeur à écho utilisé en 1991 (BioSonics modèle ES 2000 ) était entièrement sous contrôle informatique. La fréquence d'émission acoustique des appareils était de $420 \mathrm{kHz}$. En rive gauche, chaque transducteur était configuré de façon à transmettre un signal efficace dans un volume elliptique de $3^{\circ} \times 10^{\circ}$ (faisceau étroit) et à recevoir les retours d'échos par ce faisceau étroit et un second faisceau large de $7^{\circ} \times 21^{\circ}$. En rive droite, chaque transducteur avait un champ d'émission efficace conique de $6^{\circ}$ (faisceau étroit) et recevait les retours d'échos à l'aide du même faisceau et d'un second faisceau plus large de $15^{\circ}$.

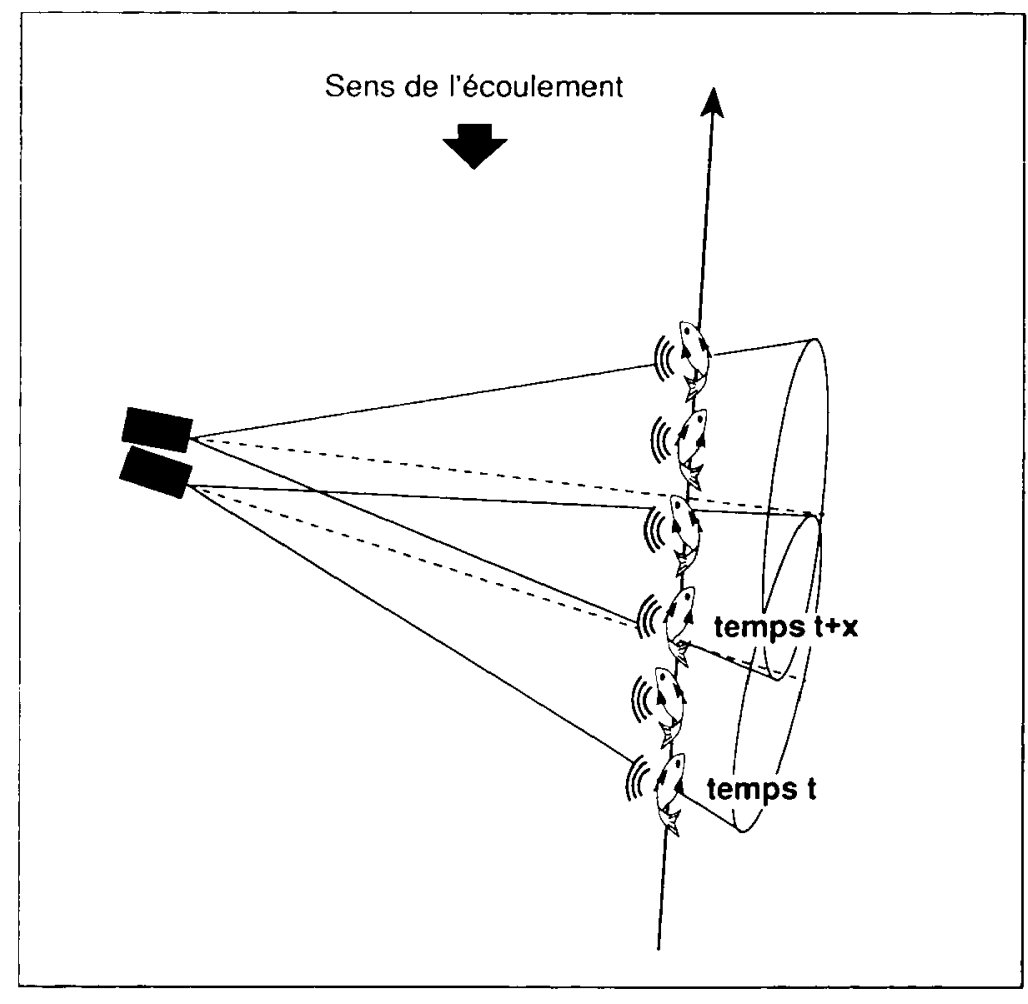

FICLLEE 2

Figure 2 : Vue en plan d'un poisson nageant dans le champ d'insonification généré par deux transducteurs à double faisceau, en couple.

Figure 2 : Plan view of a fish swimming in the ensonified field produced by paired dual-beam transducers. 


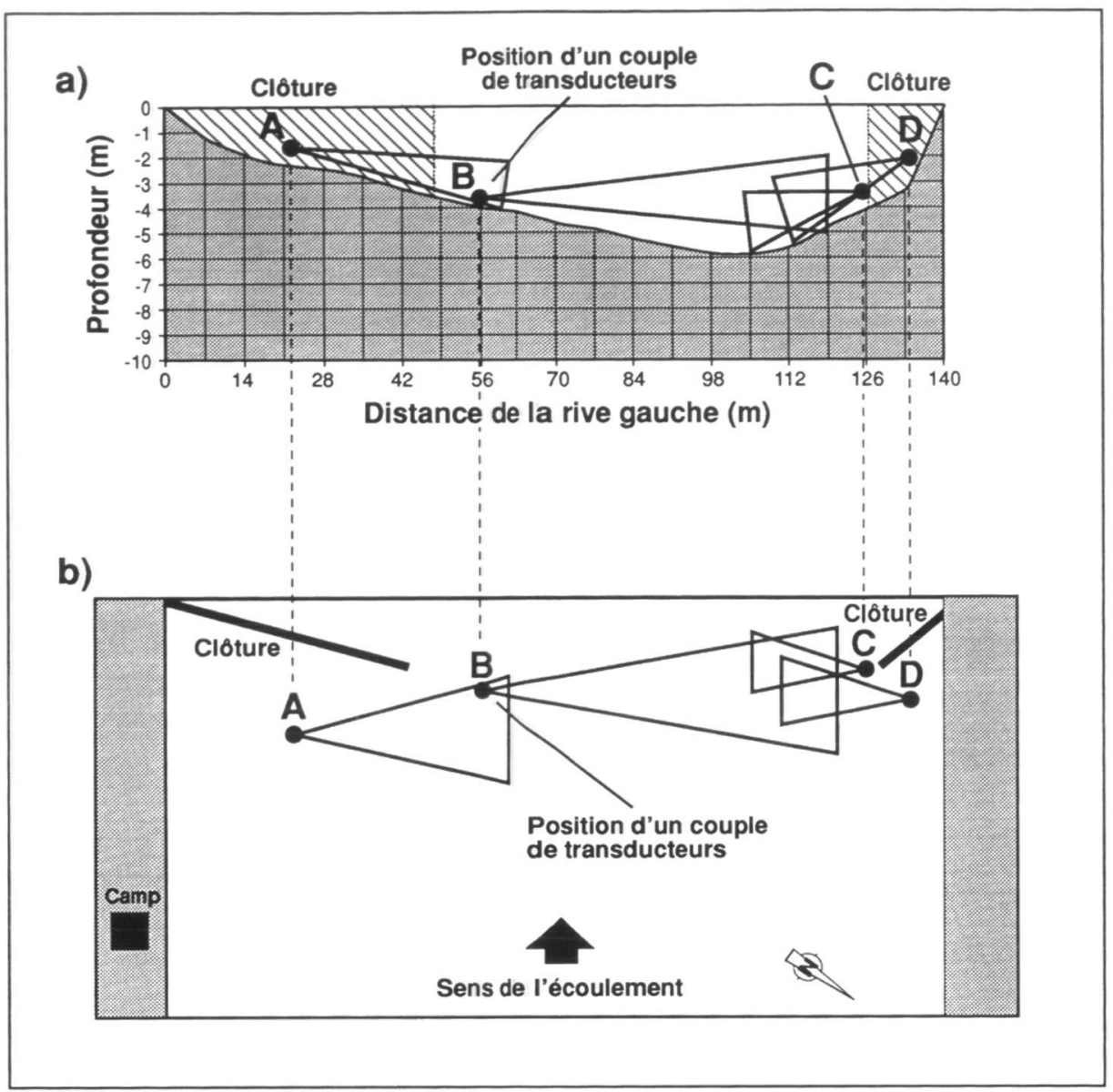

FIGURE 3.

Figure 3: Vue en coupe (a) et en plan (b) de la couverture des champs d'insonification dans la rivière Moisie, en 1991.

Figure 3 : Cross-sectional (a) and plan (b) views of the ensonified field coverage in the Moisie River, in 1991.

Les données ont été recueillies sans interruption majeure, $24 \mathrm{~h}$ sur $24 \mathrm{~h}$, de la fin juin jusqu'au début du mois de septembre. Une calibration complète des appareils a été réalisée en laboratoire, avant le début de chaque campagne, et vérifiée à trois reprises durant la période d'échantillonnage à l'aide d'une balle de ping-pong dont la taille acoustique est connue $(-42 \mathrm{~dB})$. Les appareils ont été ajustés de façon à détecter uniquement les poissons d'une taille supérieure à $25 \mathrm{~cm}$ en vue latérale, soit $-32 \mathrm{~dB}$ à la bordure des faisceaux (DAHL, 1982). Une valeur de -6dB a été ajoutée afin de compenser pour l'atténuation du niveau sonore lorsque les poissons ne voyagent pas de façon parfaitement perpendiculaire avec les transducteurs, portant l'ajustement à $-38 \mathrm{~dB}$.

L'utilisation d'un filet piège et d'une caméra vidéo sous-marine a contribué à valider le décompte des poissons détectés avec les appareils hydroacoustiques et à déterminer la composition des espèces présentes dans le secteur d'étude. Le filet piège était situé à environ un kilomètre en amont du site. La pêche fut réalisée selon un plan stratifié (FRONTIER, 1982) comportant quatre séquences d'échantillonnage de quatre à six jours consécutifs, lesquelles correspondaient au début, au pic, à la décroissance et à la fin de la montaison du saumon atlantique. La caméra (de marque Sony), pour sa part, fut introduite dans un boîtier d'aluminium étanche et installée au bout de la clôture située sur la rive gauche. Les bandes vidéo, de type VHS, ont été enregistrées en continu chaque jour entre 07 h00 et 20 h00 pour une période de 10 jours durant le pic de la migration, en 1990. 


\section{RÉSULTATS}

La majorité des poissons remontant la rivière Moisie étaient des saumons atlantiques adultes. Les autres espèces répertoriées, d'une taille supérieure à $25 \mathrm{~cm}$, étaient l'anguille d'Amérique (Anguilla rostrata), la lamproie marine (Petromyzon marinus), le meunier noir (Catostomus commersoni) et l'omble de fontaine (Salvelinus fontinalis), lesquelles constituent $19,1 \%$ des observations tirées du visionnement des bandes vidéo et $5,3 \%$ des captures au filet piège (tableau I).

Tableau I: Espèces de poissons d'une taille supérieure à $25 \mathrm{~cm}$ observées par caméra vidéo et capturées avec le filet piège dans la rivière Moisie, en 1990 et 1991.

Table 1 : Fish species larger than $25 \mathrm{~cm}$ recorded on video and captured in a trapnet on the Moisie River, in 1990 and 1991.

\begin{tabular}{|c|c|c|c|}
\hline Espèce & $\begin{array}{c}\text { Caméra vidéo } \\
\text { (nombre de) } \\
\text { (poissons par heure) }^{1}\end{array}$ & $\begin{array}{r}\text { Fil } \\
\text { (cap } \\
\text { (unit }\end{array}$ & $\begin{array}{l}\text { t piège } \\
\text { ures par) } \\
\text { d'effort) }\end{array}$ \\
\hline Anguille d'Amérique & $(3,4 \%)$ & & - \\
\hline Lamproie marine & - & 0,45 & $(5,3 \%)$ \\
\hline Anguille ou lamproie & $0,02 \quad(2,2 \%)$ & & - \\
\hline Meunier noir & $0,09 \quad(10,1 \%)$ & & - \\
\hline Omble de fontaine & $0,03 \quad(3,4 \%)$ & & - \\
\hline Saumon atlantique & $0,72 \quad(80,9 \%)$ & 8,11 & $(94,7 \%)$ \\
\hline Total & $0,89(100,0 \%)$ & 8,56 & $(100,0 \%)$ \\
\hline
\end{tabular}

(1) Données recueillies durant le jour entre $07 \mathrm{~h} 00$ et $20 \mathrm{~h} 00$.

(2) L'unité d'effort est équivalente à une journée de pêche avec le filet piège.

Les saumons atlantiques de la rivière Moisie sont des poissons dont la longueur totale a été évaluée selon les captures au filet piège à $87,2 \pm 12,5 \mathrm{~cm}(\min$. $-\max .=45,0-121,0 \mathrm{~cm})$. $\mathrm{La}$ taille acoustique des cibles enregistrées vers l'amont variait entre $-33,4$ et $-20,4 \mathrm{~dB}$.

Le visionnement des bandes vidéo démontre qu'ils nagent à une distance de $32 \pm$ $11 \mathrm{~cm}$ (min. - max.=15-80 cm) au-dessus du lit de la rivière. La distribution horizontale des cibles détectées par le sondeur à écho indique aussi que la grande majorité des saumons atlantiques n'empruntaient pas le chenal de la rivière pour migrer vers l'amont (fig. 4). Ils se déplaçaient principalement en zone peu profonde à proximité de la rive gauche dans un corridor où les vitesses d'écoulement étaient inférieures à $1 \mathrm{~m} / \mathrm{s}$ environ.

La figure 5 montre que les décomptes acoustiques de poissons sont consistants avec les captures au filet piège. Le nombre de poissons détectés en direction vers l'amont, avec l'équipement hydroacoustique, était significativement corrélé $(r=0,8538 ; \mathrm{P}<0,005)$ avec les captures de saumons atlantiques dans le filet piège. On note une meilleure corrélation des captures avec les décomptes de poissons se déplaçant vers l'amont lorsqu'ils sont combinés avec ceux dont la direction était indéterminée $(r=0,9475 ; \mathrm{P}<0,0005)$, laquelle est hautement significative.

Les observations suggèrent en effet que la plupart des poissons dont la direction était indéterminée glissaient tranquillement vers la rive gauche dans le champ du transducteur aval après avoir passé le bout de la clôture. Ce phénomène a été observé pendant toute la durée de l'étude alors qu'une forte proportion des saumons atlantiques en montaison étaient détectées entre les transducteurs et l'extrémité de la clôture de déviation (fig. 4). Cette situation a été rétablie vers la fin de l'étude avec l'installation d'un court filet en monofilament (maille étirée de $5,1 \mathrm{~cm}$ ) dans le champ d'insonification, au bout de la clôture, à une distance minimale de $10 \mathrm{~m}$ des transducteurs, qui obligeait les poissons à se déplacer parallèlement à la rive et perpendiculairement au couple de transducteurs $\mathrm{A}$. Ce dispositif n'apporte pas de complication acoustique puisqu'il produit de faibles valeurs d'indice de réflectivité. 


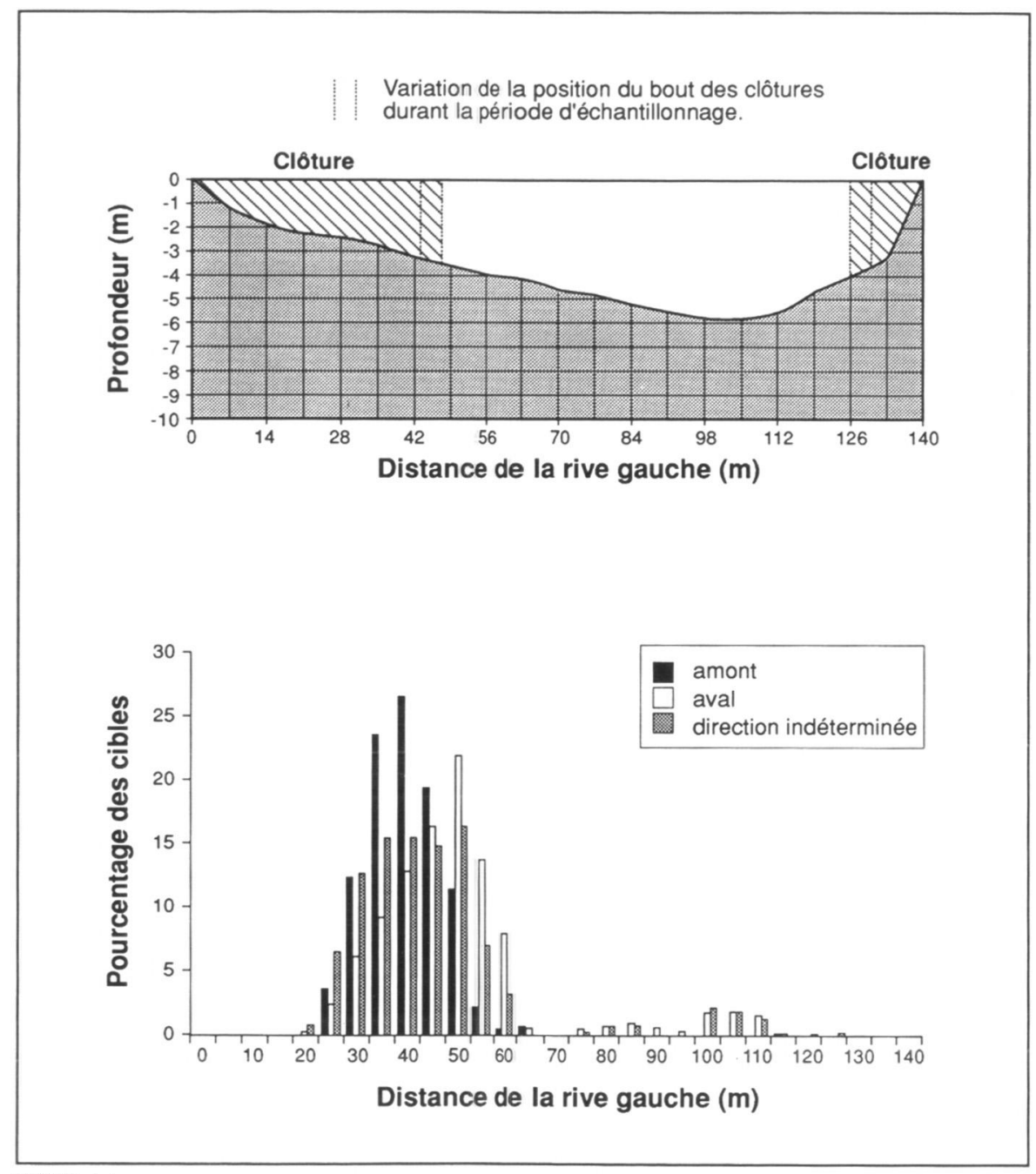

FIGURE 4.

Figure 4 : Distribution horizontale des cibles détectées dans la rivière Moisie, en 1991.

Figure 4 : Horizontal distribution of targets detected in the Moisie River, in 1991. 


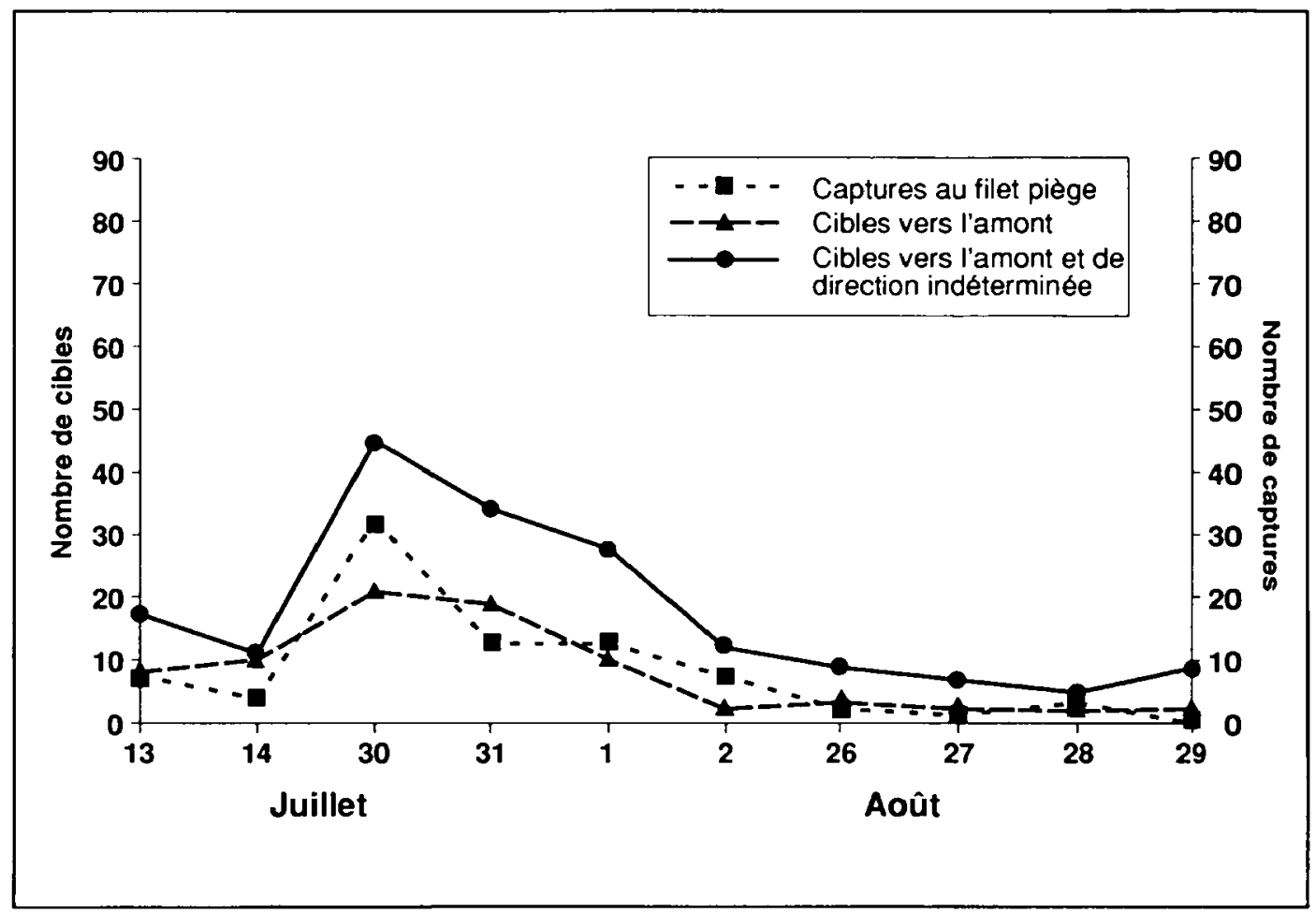

FIGURE 5.

Figure 5 : Relation entre le décompte des poissons détectés par hydroacoustique et les prises journalières de saumons atlantiques au filet piège, dans la rivière Moisie, en 1991.

Figure 5 : Relationship between counts of fish detected by hydroacoustic and daily catches of Atlantic salmons in a trap-net, in the Moisie River, in 1991.

Enfin, les résultats obtenus sur l'activité journalière des poissons indiquent qu'environ $70 \%$ des poissons en migration vers l'amont ont été détectés entre 20 h00 et 08 h00, avec des pics observés au crépuscule et avant l'aube. Dans la rivière Moisie, le saumon atlantique se déplacerait donc surtout durant la nuit.

\section{DISCUSSION - CONCLUSION}

La présente étude est, à notre connaissance, la première portant sur l'évaluation d'un stock de saumons atlantiques anadromes avec l'application de la technique de sondage par écho à double faisceau, en couple. Elle a permis de mieux connaître le comportement de ce poisson lorsqu'il se déplace en rivière, et a contribué à expérimenter un dispositif d'échantillonnage nouveau pour évaluer les stocks en migration potamique.

Les forces de cibles mesurées $(-33,4$ à $-20,4 \mathrm{~dB})$ correspondent théoriquement à des poissons dont la longueur totale est d'environ 25 à $120 \mathrm{~cm}$ (LOVE, 1971; DAHL, 1982). Les saumons atlantiques capturés dans les filets pièges possédaient des longueurs totales variant entre 45 et $121 \mathrm{~cm}$. Ceci suggère que la majorité des cibles détectées possédaient la taille de saumons atlantiques adultes, mais qu'un certain nombre de cibles plus petites ont également été détectées.

Les bandes vidéo enregistrées en période diurne montrent que le saumon atlantique se déplace près du lit de la rivière. II n'y a pas eu d'enregistrement effectué durant la nuit. II se peut que l'espèce adopte un comportement différent en période nocturne. Des transducteurs installés en projection verticale dans l'un des corridors de migration permettraient de vérifier la position des poissons dans la colonne d'eau pendant la nuit. 
Les résultats mettent en évidence que les saumons atlantiques remontent la rivière Moisie en longeant la rive. L'étude par hydroacoustique des stocks de saumons rouges (Oncorhynchus nerka) de la rivière Kenai, en Alaska, a donné des résultats similaires (KING et TARBOX, 1989), alors que les saumons quinnats (Oncorhynchus tshawytscha) de la rivière Klamath, en Californie, utilisent les zones les plus profondes dans le haut de l'estuaire pour effectuer leur déplacement (HARTE et JOHNSTON, 1989). La majorité des saumons rouges du fleuve Fraser, au Canada, se déplacent au centre du cours d'eau dans la zone d'échantillonnage qui comporte une profondeur relativement uniforme sur toute la largeur (LEVY et al., 1991). Le comportement des poissons migrateurs diffèrerait donc selon l'espèce étudiée et les différents secteurs d'une rivière.

Les vitesses d'écoulement influencent le comportement du saumon (BANKS, 1969). Dans la rivière Moisie, les saumons atlantiques en montaison étaient concentrés en rive gauche à l'intérieur d'une zone où les vitesses d'écoulement étaient généralement inférieures à $1 \mathrm{~m} / \mathrm{s}$. En rive droite, les vitesses étaient beaucoup plus élevées. Ces résultats suggèrent que le saumon atlantique recherche des conditions de confort où les vitesses d'écoulement sont plus faibles pour remonter le cours d'eau. Les poissons revenaient rapidement vers le rivage après avoir contourné le dispositif d'évitement mis en place pour favoriser leur passage dans la zone optimale de détection. GAUDET (1990) a démontré que les saumons du genre Oncorhynchus sp. avaient également tendance à se déplacer plus près des berges dans les rivières où l'écoulement était rapide.

Les données recueillies montrent que le saumon atlantique nage près du lit de la rivière. Cette observation indique que l'irrégularité dans le profil du lit de la rivière peut entraîner une sous-estimation des décomptes de poissons qui se déplacent dans les creux du lit, zone que les appareils à projection horizontale ne peuvent pas échantillonner. Comme l'ont souligné BERNING et al. (1987), le profil uniforme du lit de la rivière doit être considéré comme étant un élément critique dans la sélection du site d'étude.

Par ailleurs, mentionnons que les endroits où les poissons voyagent dans toutes sortes de directions peuvent occasionner de grandes difficultés à interpréter les résultats obtenus avec les sondages par écho. Les estuaires, où la remontée des poissons est ponctuée d'incursions successives vers l'amont et vers l'aval, avec le jeu des marées (HARTE et JOHNSTON, 1989), et les fosses de repos dans lesquelles les poissons effectuent fréquemment des mouvements circulaires, sont des exemples où l'installation d'équipements hydroacoustiques est à éviter. Ceux-ci doivent être placés dans des secteurs que traversent rapidement les poissons sans s'arrêter.

Ces quelques indications suggèrent de bien connaître les caractéristiques du site d'échantillonnage et le comportement du poisson, pour effectuer efficacement le suivi des stocks avec l'usage des techniques hydroacoustiques. Ces informations sont déterminantes de la position et de l'orientation des transducteurs, par rapport au profil du lit de la rivière et aux vitesses d'écoulement, qui sont des facteurs d'une très grande importance à considérer avec précaution dans l'étude des poissons migrateurs par sondage à double faisceau, en couple.

La technique de sondage par écho permet difficilement de distinguer les espèces autrement que par leur taille ou leur comportement. Pour cette raison, il est essentiel de valider les résultats au moyen des techniques traditionnelles de pêche ou d'autres technologies modernes, comme le système vidéo employé dans cette étude. Les captures au filet piège ainsi que les observations provenant de la caméra sous-marine démontrent que la majorité des poissons détectés en direction amont avec les appareils hydroacoustiques étaient des saumons atlantiques adultes dans la rivière Moisie. De plus les captures de saumons atlantiques étaient fortement corrélées avec les décomptes acoustiques de poissons.

Une contrainte de la technique de sondage à double faisceau est le niveau de bruit de fond généré dans l'environnement fluvial qui, dans le cas d'un grand cours d'eau comme la rivière Moisie, peut augmenter avec la variation des niveaux d'eau surtout lorsque les faisceaux sont accolés à proximité du lit de la rivière. Le niveau de bruit mesuré dans la présente étude atteint des valeurs qui pourraient masquer la présence de plus petits poissons, mais pose peu de contraintes à la détection des saumons atlantiques adultes. Le rapport entre l'amplification du signal transmis par les appareils et le niveau de bruit mesuré 
peut contribuer à solutionner ce problème. Toutefois, des précautions doivent être prises afin de bien positionner les faisceaux par rapport au lit de la rivière et à la surface de l'eau, afin de réduire le bruit de fond tout en optimisant la couverture des strates de profondeurs propices à la détection des poissons étudiés.

\section{REMERCIEMENTS}

L'étude du saumon de la rivière Moisie a été réalisée grâce au soutien d'HYDROQUÉBEC, dans le cadre de l'aménagement du complexe hydroélectrique SainteMarguerite. Nos remerciements s'adressent également à Monsieur Gilles SHOONER, pour sa revue critique du manuscrit.

\section{BIBLIOGRAPHIE}

BANKS J.W., 1969. A review of literature on upstream migration of adult salmonids. J. Fish. Biol., 1, 85-136.

BERNING R.L., MESIAR D.C., GAUDET D.M., 1987. Sonar enumeration of migrating fish in the Noatak River, 1984. Alaska Department of Fish and Game, Division of Commercial Fisheries, Informational leaflet 262, $67 \mathrm{p}$.

DAHL P.H., 1982. Analysis of salmonid target strength and Doppler structure for riverine sonar application. M.S. Thesis. University of Washington, Seattle, WA, U.S.A.

EHRENBERG J.E., 1982. A review of in situ target strength estimation techniques in O. Nakken, S.C. Venema (eds.). Symposium on Fisheries Acoustics, selected papers of the ICES/FAO Symposium on Fisheries Acoustics, Bergen, Norway, 21-24 June 1982, p. 85-90, FAO Fish. Rep. (300), 331 p.

EHRENBERG J.E., 1984. Principles of dual-beam processing for measuring target strengths. Technical Note \#41, BioSonics inc., Seattle, Washington, $4 \mathrm{p}$.

FRONTIER S., 1982. Stratégies d'échantillonnage en écologie. Masson, Paris et les Presses de l'Université Laval, Québec, 494 p.

GAUDET D.M., 1990. Enumeration of migrating salmon populations using fixed location sonar counters. Rapp. P.-v. Réun. Cons. int. Explor. Mer., 189, 197-209.

HARTE M.K., JOHNSTON S.V., 1989. Final report of hydroacoustic monitoring on the Klamath River, 1986 to 1988 (including annual report for the summer 1988). Submitted to California Department of Fish and Game. Sacramento, California, USA, $56 \mathrm{p}$. and appendices.

KING B.E., TARBOX K.E., 1989. Upper Cook Inlet salmon escapement studies, 1987. Alaska Department of Fish and Game, Juneau, Alaska, USA, Technical Fishery Report 89-11.

LEVY D.A., NEALSON P.A., CHENG P., 1991. Fixed-aspect hydroacoustic estimation of Fraser River sockeye salmon abundance and distribution at Mission, B.C., in 1986. Pacific Salmon Comm. Tech. Rep. N 4, 30 p.

LOVE R.H., 1971. Dorsal aspect target strength of an individual fish. J. Acoustic Soc. Amer, 49, 816-823.

MATHISEN O.A., 1980. Acoustic stock assessment in T. Backiel, R.L. Welcome (eds.). Guidelines for sampling fish in inland waters, p. 115-141, EIFAC Tech. Pap. (33), $176 \mathrm{p}$.

THORNE R.E., 1983. Hydroacoustics in L.A. Nielsen, D.L. Johnson (eds.). Fisheries techniques, p. 239-259, American Fishery Society, Bethesda, Maryland, 468 p. 